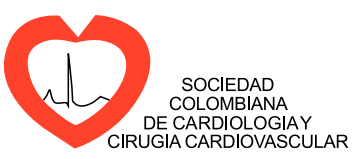

\title{
EDITORIAL
}

\section{And... where is the patient?}

\section{Y... ¿dónde está el paciente?}

\section{Darío Echeverri}

\author{
Revista Colombiana de Cardiología
}

Received 19 May 2016; accepted 19 May 2016

Available online 18 June 2016

\begin{abstract}
"It has cost me a lot to become a physician, and to continue to be one costs me unwearying and continuous vigilance; the stalking of an implacable introspection...",
\end{abstract}

(Escardó)

A propos of the 200 years of the stethoscope, the word comes from the Greek, stethos (chest) and scopos (exam). Ever since man began studying human physiology and the physical characteristics associated with various diseases, the heart sounds, and those of the surrounding organs, have been crucial indicators in the patient's exam. The first stethoscope was invented in 1816 by the young French physician, René Théophile Hyacinthe Laënnec (1781-1826), who studied medicine in Paris with several famous physicians, including Dupuytren and Jean Nicolas Corvisart Desmarets.

One day, in the fall of 1816, Laënnec was scheduled to examine a young woman with general symptoms of a diseased heart. Laënnec needed to listen to the chest of the somewhat overweight woman, but he considered it to be a bit inappropriate and insufficient to place his ear on her chest and listen directly to the heart sounds. He therefore rolled up a sheet of paper in the shape of a tube, and placed one end on the patient's chest. The tube magnified the sound, and Laënnec discovered that he could easily hear the heart and lung sounds by placing his ear on the open

DOI of original article: http://dx.doi.org/10.1016/j.rccar. 2016.05.005

E-mail address: decheverri@cardioinfantil.org

URL: http: //www.revcolcard.org/. end. The rolled up piece of paper was soon replaced by a hollow wooden tube. On August 19, 1819, he published his masterpiece, "De l'auscultation médiate", a two volume book which caused a great stir in the world of medicine. Known as the father of clinical auscultation, Laënnec is considered to be one of the greatest physicians of all times.

As we celebrate the 200 years since the discovery of the stethoscope, this may be the instrument that today brings the physician closest to the patient, which gives it a unique and unequaled identity. Therefore, it is worthwhile to pause and reconsider our professional medical essence, and even more as cardiologists. The physician's mission is to preserve and restore the patient's health. However, medical problems are not always the same: they change with the changing culture, vary from region to region; they are dynamic, belonging to a given time in history, in a given society. In addition, we are in a time of dizzying change. Diseases, like societies, seem to transmute. For many centuries, man's and society's evolution has been constant and persistent, but never had such a swift rhythm been reached, with such an abundance of knowledge, nor had such solid scientific achievements been reached, with such an impact on health.

The increasing growth in population and its basic needs has come hand in hand with important advances in science and technology, which obligates the preparation of professionals who are able to equally join a scientific, technical and social movement, which, while representing a problem, as with all problems, implies an opportunity. The new modalities of the physician's professional exercise have produced, worldwide, a fracture in the old relationships 
between the physician and the ill person, and between the physician and the community. This phenomenon carries the risk of dehumanizing and depersonalizing medicine.

Likewise, never has there been so much interest in the human being as in today's medicine; and what we can see is a fading of the physician's personality and his direct relationship with the patient within institutional medicine. Certain tendencies try to turn us into "specialized technicians". As strange as it may seem, many of us feel a fascination with being one, with fulfilling the technician's functions, as such, due to the mirage this technical solution offers to study, research, treatment and professional activity. This orientation of medical practice, brought about by the pressure of modern technology, unfortunately leads to the physician sacrificing contact with the patient, with the human being, and losing the meaning of the profession, because the technician does not go beyond the procedure. He becomes an efficient, necessary executor, but nothing more. He does not consciously advance toward a total knowledge, to an integral practice and toward the understanding of the patient's humanity. Similarly, never before has there been so much interest in human beings as there is in today's medicine, and there is a feeling that the personality of the physician and their direct relationship with the patient within insitutional medicine is fading.

The physician's specialization and superspecialization have been an inevitable result of this evolution. They overcome the logical limitations of attempting to encompass everything in a single mind; they increase the depth and detail of partial aspects. The human tendency to project itself in reduced fields in order to cope with the pressure is normal. Specialization favors and needs teamwork. However, we can fragment knowledge, but not the individual.
It is not unusual for patients and their families to simply need to engage in a dialogue, or to relate personal issues, fears and hopes. They don't necessarily need complementary exams, implantation of devices, complex surgeries or multidisciplinary interventions. Perhaps what they want is to come closer and trust in the medical professional, rather than distance themselves.

So, therefore, medical schools should not only prepare the "physicians" needed to cover the physical needs of the population, but should also conserve and communicate the individual and collective moral values, and seek the best social environment. In an adequate education and an appropriate example, the young physician will find a stimulus to his service vocation and a firm adhesion to the scientific method which will allow him to acquire the necessary knowledge, be able to understand and take advantage of scientific research, and assimilate the continued advance in knowledge. Education will also have to generate techniques, abilities and skills for the correct application of the new technologies applied in the diagnosis and treatment of so many diseases, modulate the mind and the exercise of the profession in order to understand the human being and his environment, promote collective betterment, and favor social evolution.

Our patients are not easily summarized in medical practice guidelines, nor in numbers, scales, or risk scores. We should think again of the essence of the exercise of medicine, and, with the aid of the stethoscope, get closer to the patient and get to know him in an integral fashion. This, together with the technological advances in medicine, clinical indicators, biomarkers and measurements, among many other available tools, will promote a modern, efficient, rational and ethical professional practice, in accord with the needs of the population. 